\title{
Micromagnetic Simulation of Thermal Generation of Spin Waves Using MuMax3
}

\author{
J. Woźny*, R. Gozdur, B. Guzowski and Ł. Bernacki \\ Department of Semiconductor and Optoelectronic Devices, Lodz University of Technology, \\ Wolczanska 211/215, 90-924 Lodz, Poland
}

\begin{abstract}
In this paper the micromagnetic simulation of thermal generation of spin waves in permalloy is shown. The simulation was carried out with MuMax3 software. The relationship between the value of external applied field and frequency of the generated spin waves, as well as the influence of sample temperature on spin waves amplitude were investigated and they are described. Thermal generation of spin waves was simulated for room temperatures.
\end{abstract}

DOI: 10.12693/APhysPolA.137.737

PACS/topics: spin wave, MuMax3, thermal generation

\section{Introduction}

More than 10 years ago the spin Seebeck effect (SSE) was reported [1]. This effect was measured by means of the inverse spin Hall effect (ISHE) where spin current is converted to the charge current of density $\boldsymbol{J}_{c}=\theta_{S H}\left(\frac{2 e}{\hbar}\right) \boldsymbol{J}_{S} \times \boldsymbol{\sigma}$, where $\theta_{S H}$ is the spin Hall angle, $\boldsymbol{J}_{C}$ is the charge current, $\boldsymbol{J}_{S}$ is the spin current, $e$ is the electronic charge, $\hbar$ is reduced Plank constant, and $\boldsymbol{\sigma}$ is the spin polarization. Due to the development of ultralow power electronics $[2,3]$ recently it appeared that the SSE+ISHE can be considered as a new way of energy harvesting technique [4-7] enabling conversion of wasted heat to useful electric energy. Spin caloritronics especially with the use of low cost and well developed permalloy [8] or yttrium iron garnet (YIG) which has extremely low damping factor can be useful in new generation of temperature sensors [9].

To understand the magnetic nanostructures better it is important to model the thermal generation of spin waves (SWs). In this paper we would like to discuss the possibility of the use of MuMax3 software [10-14] to investigate this phenomenon. The MuMax3 software is widely used for micromagnetic simulations. However, it has not been shown if it could be used for simulation of SWs at room temperatures. There are few micromagnetic simulations presented by the authors of MuMax3 $[10,15]$ in which thermal effects have been taken into account. However, none of them concerns the SWs transport.

\section{Simulated geometry}

A simulation was performed for a nanostripe of permalloy of $6000 \times 100 \times 5 \mathrm{~nm}^{3}$. The grid cell size is $2 \times 2 \times 5 \mathrm{~nm}^{3}$. The structure is shown in Fig. 1 . On the both ends of the structure there are damping regions with $L_{D}=23.53 \mathrm{~nm}$. An external magnetic field

\footnotetext{
* corresponding author
}

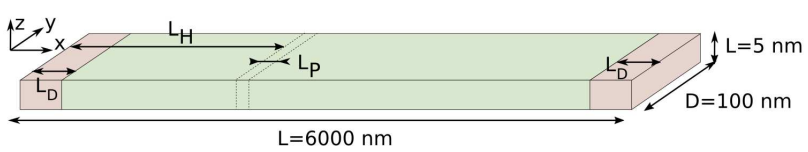

Fig. 1. The simulated permalloy geometry.

$\boldsymbol{B}=\left[B_{x}, B_{y}, B_{z}\right]=\left[B_{X 0}, 10^{-6}, 10^{-6}\right] \mathrm{T} \quad$ was applied. The $B_{X 0}$ changes from $1 \times 10^{-4}$ to $0.5 \mathrm{~T}$. The parameters of permalloy are: saturation magnetization $M_{\mathrm{SAT}}=860 \times 10^{3} \mathrm{~A} / \mathrm{m}$, the Landau-Lifshitz damping constant $\alpha=0.01$, and exchange stiffness $A_{\mathrm{EX}}=1.3 \times 10^{-11} \mathrm{~J} / \mathrm{m}[15]$.

\section{Verification of SWs frequency calculation in MuMax3}

First, at zero temperature, it was verified and confirmed whether the generated SWs frequency agrees with analytical predictions [15]. The constant external magnetic field $B$ was used as DC-bias to order the investigated system. The Heaviside pulse of external magnetic field was then applied to a slice of thickness $L_{P}=23.53 \mathrm{~nm}$ located at $L_{H}=588 \mathrm{~nm}$ distance from the left side. This pulse was used to introduce a local disturbance of spins and to cause the SWs to appear. It is defined as:

$$
B_{Y}(t)=1 \times 10^{-6}-B_{Y 0} \theta\left(t-0.5 \times 10^{-9}[\mathrm{~s}]\right),
$$

where $\theta(t)$ is the Heaviside step function delayed by $0.5 \mathrm{~ns}$, and $B_{Y 0}$ is the amplitude. The Heaviside pulse introduces local disturbance of magnetization vector. An exemplary distribution of magnetization vector $\mathbf{m}$ is shown in Fig. 2. It depicts spatial distribution of $m$ for $t=0.89 \mathrm{~ns}$, that is $0.39 \mathrm{~ns}$ after the magnetic field pulse was applied.

The wavelength of a SW was calculated by means of fast Fourier transformation (FFT) analysis of spatial distribution of magnetization vector, that is shown in Fig. 2. The domain between points $\mathrm{A}$ and $\mathrm{B}$, that is outside the slice, where the Heaviside pulse was applied, have been 


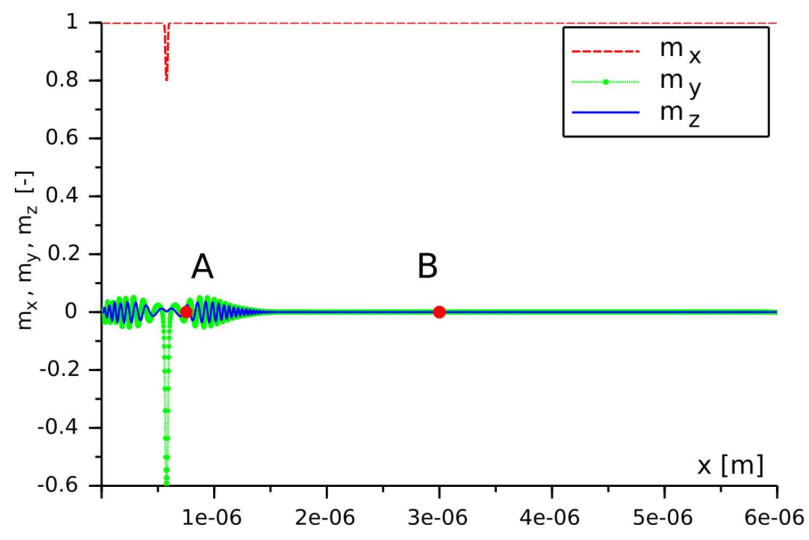

Fig. 2. Normalized magnetization vector $\boldsymbol{m}$ distribution for $t=0.89 \mathrm{~ns}, B_{X 0}=500 \mathrm{mT}, T=0 \mathrm{~K}$.

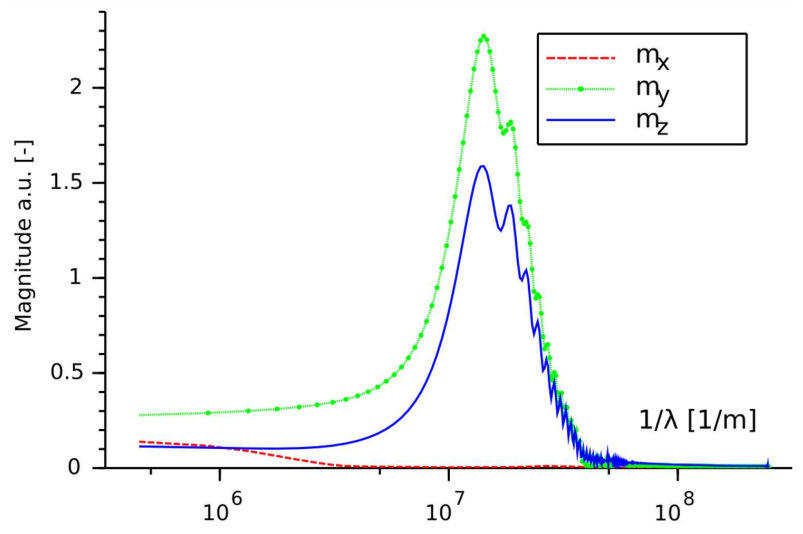

Fig. 3. FFT analysis of spatial $\boldsymbol{m}$ distribution for $t=$ $0.89 \mathrm{~ns}, B_{X 0}=500 \times 10^{-3} \mathrm{~T}, B_{Y 0}=500 \mathrm{mT}, T=0 \mathrm{~K}$.

considered. The results of the FFT analysis are shown in Fig. 3. From that, one can easily retrieve the wavelength of the SW which corresponds to the maximal value of FFT signal.

Since the wavelength is known, the analytical equation [15] to calculate expected frequency $f$ of SWs can be used.

$$
f=\frac{\mu_{0} \gamma}{2 \pi} \sqrt{H\left(H+M_{\mathrm{SAT}}\right)+\frac{M_{\mathrm{SAT}}^{2}}{4}\left(1-\mathrm{e}^{-2 k d}\right)},
$$

where $H$ is the external magnetic field, $\mu_{0}$ is the magnetic permeability of vacuum, $\gamma$ is the gyromagnetic ratio, $k$ is the wave vector, and $d$ is the thickness of a sample.

Frequency of a SW can also be directly retrieved from analysis of a time evolution of magnetization vector $\boldsymbol{m}$ by means of the FFT analysis. An exemplary evolution of magnetization vector and the FFT results are depicted in Fig. 4.

The SWs frequency calculated from the formula and obtained directly from simulation are in good agreement as it is presented in Fig. 5 as a function of external magnetic field.

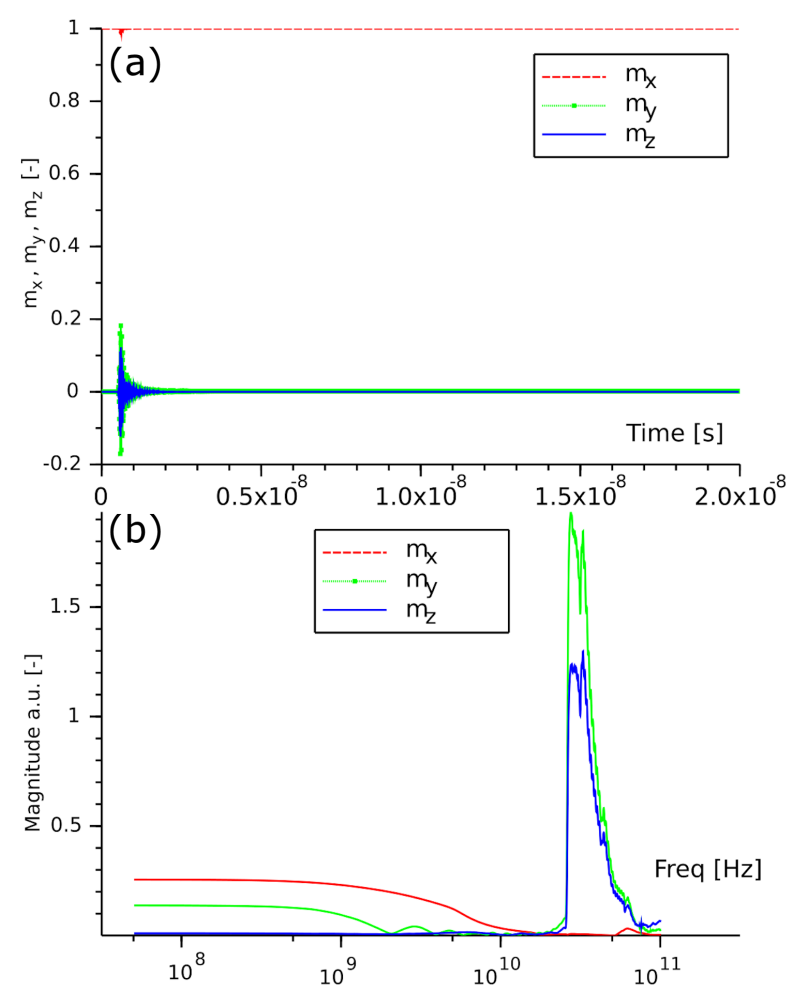

Fig. 4. (a) Magnetization vector $\boldsymbol{m}$ as function of time at $x=0.67 \mu \mathrm{m}$, (b) magnitude of the Fourier transform of signal shown in (a) $B_{X 0}=500 \times 10^{-3} \mathrm{~T}$, $B_{Y 0}=500 \mathrm{mT}, T=0 \mathrm{~K}$.

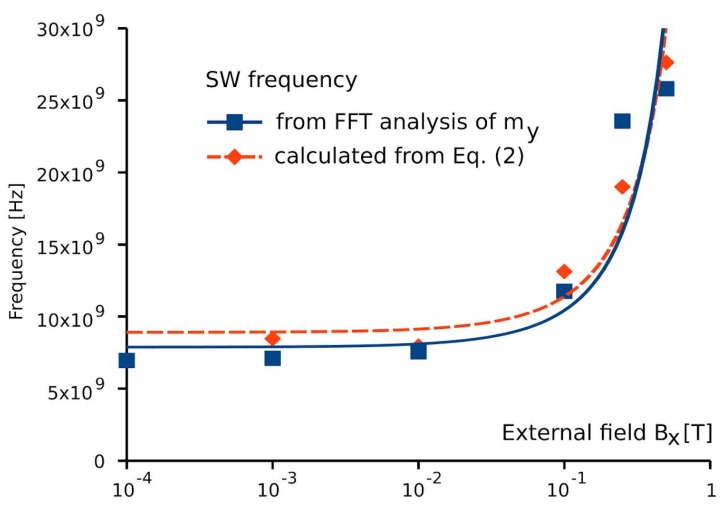

Fig. 5. Comparison of SWs frequency calculated by means of analytical formula and from FFT analysis $B_{Y 0}=B_{X}$.

\section{SWs calculation at elevated temperatures}

The simulation described in Sect. 3 was performed to verify if MuMax3 can predict SWs correctly. Since the agreement between simulation and theory was achieved it seems reasonable to use MuMax3 to simulate the generation of SWs due to non-zero temperature of the domain. MuMax3 takes into account thermalisation of magnetic 


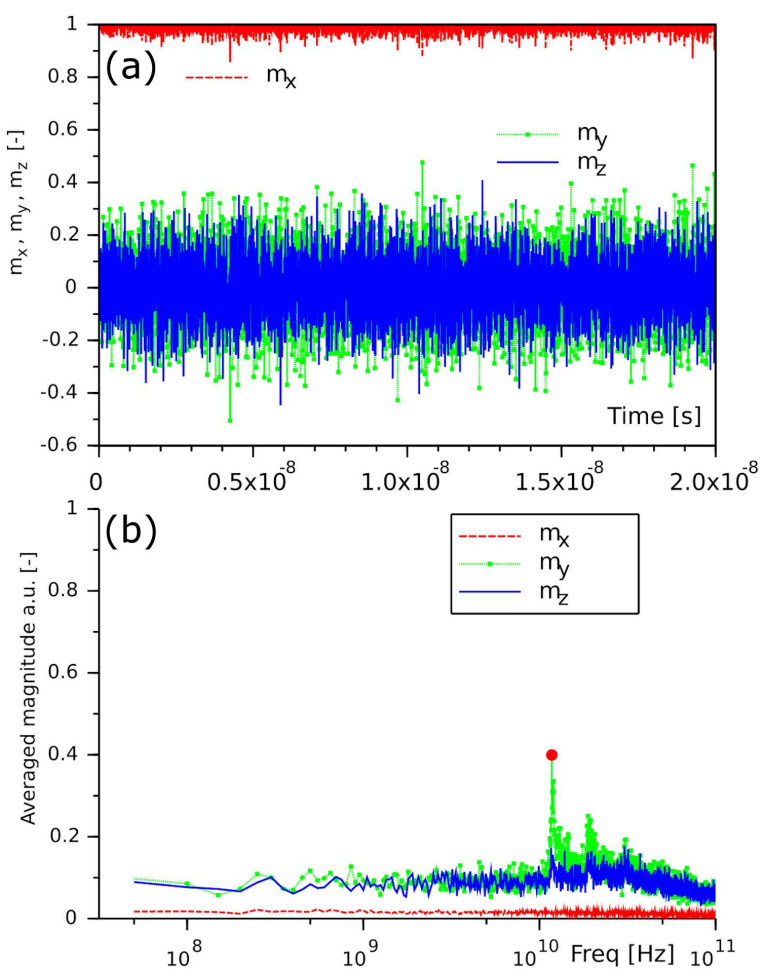

Fig. 6. (a) Magnetization vector $\boldsymbol{m}$ as a function of the time at a single location, $T=300 \mathrm{~K}$, (b) magnitude of averaged Fourier transform.

domain by means of artificial stochastic external magnetic field that leads to statistically correct distribution of magnetization vector [14]. Simulation was performed for the temperature ranging from $100 \mathrm{~K}$ to $600 \mathrm{~K}$ and for external vector $\boldsymbol{B}=\left[B_{\mathrm{EXT}}, 1 \times 10^{-6}, 1 \times 10^{-6}\right] \mathrm{T}$, $B_{\mathrm{EXT}}=\{50,100,500\} \times 10^{-3} \mathrm{~T}$. The system has been relaxed first and then the magnetization vector distribution $\mathbf{m}$ was recorded from $t=0 \mathrm{~s}$ to $t=20 \mathrm{~ns}$ with steps of $\Delta t=5 \mathrm{ps}$. An example of vector $\boldsymbol{m}$ is presented in Fig. 6a. Again, the FFT has been performed to obtain the basic frequency of the signal. Since the signal is very noisy, we have averaged the magnitude of FFT over different locations. The FFT has been performed for $\boldsymbol{m}$ recorded at 10 points uniformly distributed along $x$ direction and results are shown in Fig. $6 \mathrm{~b}$.

The frequency $f$ of SWs, as shown in Fig. 7a, does not change within the temperature range. It is caused by the fact that the saturation magnetization was assumed to be constant. The frequency corresponds with the values reported in the literature. It was reported [16] that SW frequency measured for $B_{\mathrm{EXT}}=50 \mathrm{mT}$ is $7.2 \mathrm{GHz}$ while the value $9.7 \mathrm{GHz}$ was retrieved from simulations. The amplitude (Fig. 7b) increases first with rise of the temperature, but for temperatures higher than $400 \mathrm{~K}$ and $B_{\mathrm{EXT}}=\{100,500\} \mathrm{mT}$ the amplitude of the fundamental harmonic decreases. It is caused by the fact that at high temperatures higher harmonic frequencies become more significant and energy of SW is redistributed between them.

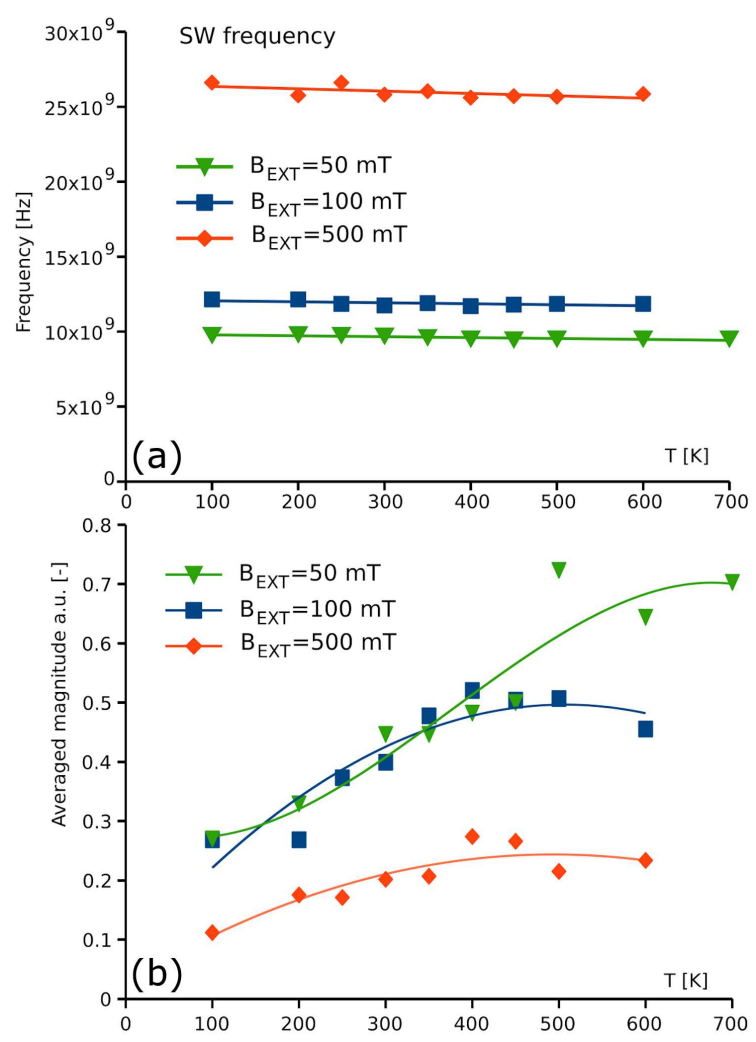

Fig. 7. (a) The frequency of SW for different temperature, (b) corresponding averaged magnitude of the SW obtained from FFT.

\section{Conclusions}

The Fourier analysis performed for the magnetization vector $\boldsymbol{m}$ revealed the dominating SW frequency, equal to $f=9.7 \mathrm{GHz}$ for $B_{\mathrm{EXT}}=50 \mathrm{mT}$. This frequency agrees with the analytical prediction and is close to experimental results. Simulations have shown that at temperatures higher than room temperature next harmonics are excited which leads to decrease of the amplitude of the 1st harmonic. In this paper it was shown that by means of the MuMax3 it is possible to obtain the thermally generated SWs.

\section{Acknowledgments}

This work was partially supported by the National Science Centre under the program "MINIATURA 2" (Project number DEC-2018/02/X/ST7/03291).

\section{References}

[1] K. Uchida, S. Takahashi, K. Harii, J. Ieda, W. Koshibae, K. Ando, S. Maekawa, E. Saitoh, Nature 455, 778, (2008).

[2] J.M. Gilbert, F. Balouchi, Int. J. Autom. Comp. 5, 334, (2008). 
[3] B. Guzowski, R. Gozdur, M. Lakomski, Metrol. Meas. Syst. 25, 235 (2018).

[4] A. Cahaya, O.A. Tretiakov, G.E.W. Bauer, IEEE Trans. Magn. 51, 0800414, (2015).

[5] A. Kirihara, K. Uchida, Y. Kajiwara, M. Ishida, Y. Nakamura, T. Manako, E. Saitoh, S. Yorozu, Nat. Mater. 11, 686 (2012).

[6] K. Uchida, M. Ishida, T. Kikkawa, A. Kirihara, T. Murakami, E. Saitoh, J. Phys. Condens. Matter 26, 389601 (2014).

[7] A. Kirihara, K. Kondo, M. Ishida et al., Sci. Rep. 6 23114 (2016)

[8] B. Guzowski, B. Guzowski, M. Hasiak, Acta Phys. Pol. A 135, 308 (2019).

[9] T. Liao, Z. Ye, J. Chen, IEEE Trans. Electron. Dev. 64, 2655, (2017).

[10] A. Vansteenkiste, J. Leliaert, M. Dvornik, M. Helsen, F. Garcia-Sanchez, B. Van Waeyenberge, AIP $A d v$. 4, 107133 (2014)
[11] L. Exl, S. Bance, F. Reiche, T. Schref, H.P. Stimming, N.J. Mauser, J. Appl. Phys. 115, 17D118 (2014).

[12] J. Leliaert, B. Van de Wiele, A. Vansteenkiste, L. Laurson, G. Durin, L. Dupré, B. Van Waeyenberge, J. Appl. Phys. 115, 233903 (2014).

[13] J. Mulkers, B. Van Waeyenberge, M.V. Milošević, Phys. Rev. B 95, 144401 (2017).

[14] J. Leliaert, J. Mulkers J. De Clercq, A. Coene, M. Dvornik, B. Van Waeyenberge, AIP Adv. $\mathbf{7}$, 125010 (2017).

[15] H. Hata, T. Moriyama, K. Tanabe, K. Kobayashi, R. Matsumoto, S. Murakami, J.-I. Ohe, D. Chiba T. Ono, J. Magn. Soc. Jpn. 39, 151 (2015).

[16] K.S. Olsson, K.M. An, X. Ma, S. Sullivan, V. Venu, M. Tsoi, J.S. Zhou, L. Shi, X.Q. Li, Phys. Rev. B 96, 024448 (2017). 